\title{
Mother and child care services 'insufficient to save lives'- HSRC report
}

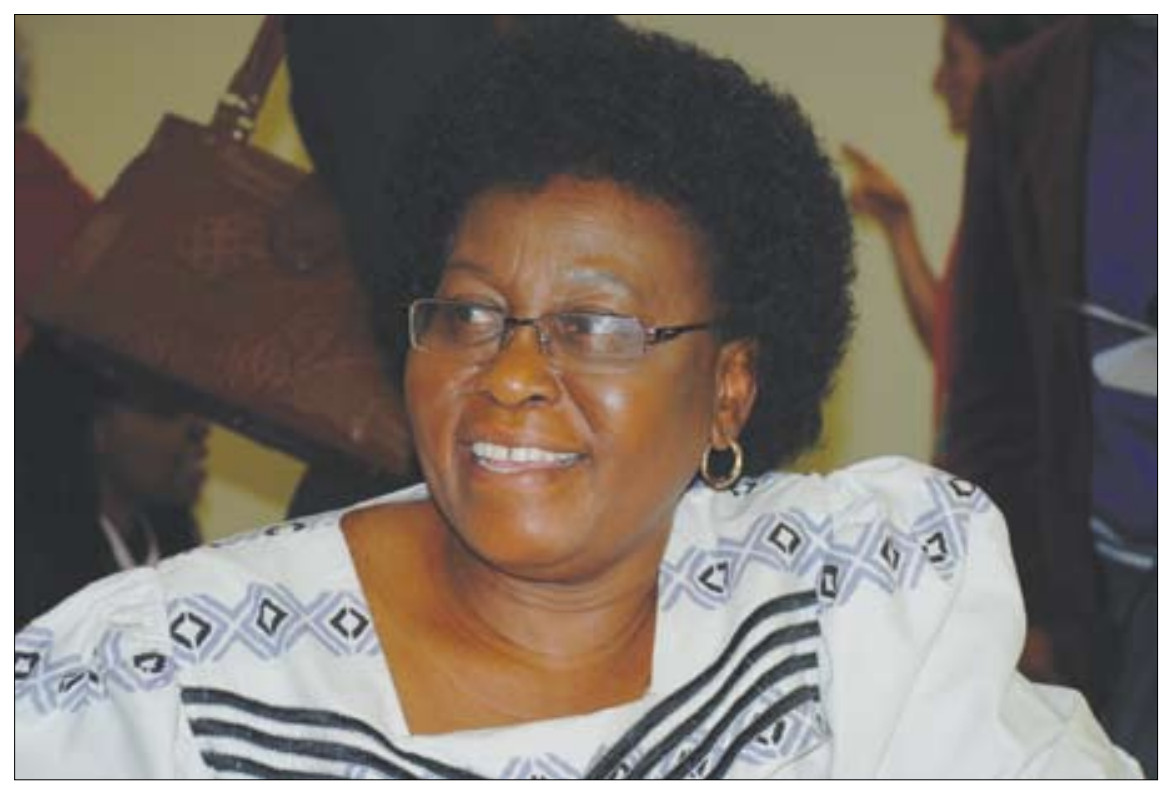

Lead investigator, Dr Olive Shisana, of the HSRC.

Picture: Chris Bateman

In spite of $77 \%$ of infants being born in hospital with the help of a skilled birth attendant, 'massive problems' with South Africa's maternal and child health services contribute to 23000 stillbirths and a neonatal mortality rate of 21 per 1000 live births yearly.

Excluding HIV, the major causes of these deaths (reported in 2008) were preterm birth complications (40\%), birth asphyxia (23\%), infections (19\%) and congenital abnormalities (10\%).

The poor quality of maternal and child health care was singled out by the Human Sciences Research Council (HSRC)'s principal investigator, Dr Olive Shisana, in presenting her team's latest household survey findings in Cape Town in mid-May.

The HSRC report said that although facilities were available and most

The HSRC report said that although facilities were available and most women had access to them,

'it appears the services provided are insufficient to save the lives of these babies'.

women had access to them, 'it appears the services provided are insufficient to save the lives of these babies'. It was 'critical' that standards for maternal health and newborn care be improved as part of the health department's 10-point plan, with more financial resources provided. Serious inadequacies included guidelines not followed, inadequate equipment and a lack of doctors at a time when they were most needed. Shisana described the problems as 'massive'.

Entitled The Health of Our Children, ${ }^{2}$ the 2008 survey of 15000 households gauged national HIV prevalence, incidence, behaviour and communication and underlined the urgency with which the new health minister, Dr Aaron Motsoaledi, has begun acting.

Seated alongside Shisana at the briefing, Motsoaledi took some comfort in hearing that by 2008 most pregnant women were using antenatal care clinics $(97 \%)$ and that three-quarters of them were receiving antiretroviral therapy (ART) and prevention of mother-tochild transmission (PMTCT) treatment. Shisana said this had translated directly into a $2.1 \%$ HIV prevalence lower than the $3.3 \%$ HIV average in the age group $0-4$, indicating that the PMTCT programme, initiated in 2006, was working. The Western Cape, with its superior infrastructure and early politically defiant PMTCT roll-out, fared best with an under 2-year-old HIV prevalence of below $1 \%$. By comparison, the figure for Mpumalanga, famous for its former AIDS denialist health care leaders, stood at $4.5 \%$.

\section{Shortcomings easily identified}

A study of adverse incidents at State sector maternity departments by the Council for Health Service Accreditation of Southern Africa (COHSASA) ${ }^{3}$ showed that the overall child mortality rates are sent soaring by easily identifiable shortcomings. among children under 2 years old,

\section{Entitled The Health of Our Children, ${ }^{2}$ the 2008 survey of 15000 households gauged national HIV prevalence, incidence, behaviour and communication and underlined the urgency with which the new health minister, Dr Aaron Motsoaledi, has begun acting.}

These included septic cases post surgery ( $8 \%)$; sets for administering blood not available when needed (4\%); uncontrolled bleeding after caesarean owing to eclampsia (12\%); mother delivered in bed; baby not breathing for 10 minutes (4\%); power failure (12\%); no doctor; baby delivered in ambulance; no oxygen and no doctor in ambulance; patient transferred (4\%); fresh stillborn baby delivered (4\%); delay in transfer; fetal distress (19\%); critically ill patient to be ventilated; no resuscitator available (4\%); and maternal deaths (12\%). factors' the survey reported that almost half a million (419 144) children in section $(8 \%)$; caesarean section delayed

Under the heading 'Contextual 


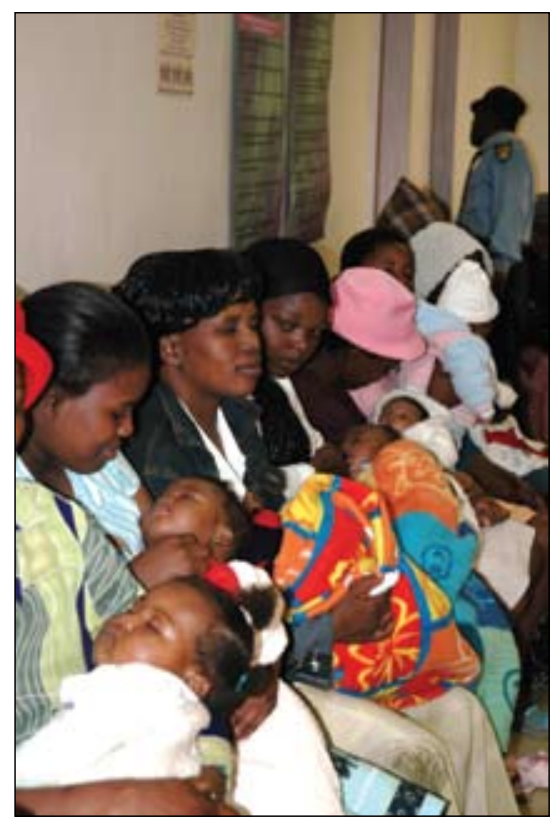

Mothers and their infants queue for check-ups at Nkandla District Hospital in deep rural KwaZulu-Natal.

Picture: Chris Bateman

South Africa are growing up without parents, mainly, but not exclusively, because of HIV. There were estimated to be approximately 3 million orphans $10.5 \%$ paternal and $3.9 \%$ maternal. The likelihood of being orphaned increased with age, with one-third of teenagers between 15 and 18 years without one parent or both parents. Most orphans were in the Eastern Cape (23.2\%), followed by KwaZulu-Natal (19.4\%), with the Northern and Western Cape having the fewest orphans. There was a decline in the number of child-headed households from $2.6 \%$ in 2005 to $1 \%$ in 2008.

\section{A study of adverse incidents at State sector maternity departments by the \\ Council for Health Service Accreditation of Southern Africa (COHSASA) ${ }^{3}$ showed that the overall child mortality rates are sent soaring by easily identifiable shortcomings.}

\section{Girls having sex with older men}

Girls aged 12 - 14 were far more likely to have had sex than boys in the same age group, with 1 in 7 girls having had sex compared with 1 in 10 boys.

Worryingly, one-quarter of girls aged 12 - 18 had most recently had sex with males who were 5 or more years older than they were. (Older males are more likely to be HIV positive.) Of the sexually active boys aged 15 - 18, more than one-quarter had had more than 2 partners in the past year as opposed to 1 in 10 girls. Condom use was an encouraging $92.1 \%$ among males and $83.9 \%$ among females.

About $8 \%$ of children reported that male teachers tried to have sex with schoolgirls, an abuse the report said should not be tolerated, recommending strong disciplining of errant teachers.

\section{Major recommendations were that the number and scope of community health workers be expanded to include high-impact but low-cost health and nutrition interventions.}

Major recommendations were that the number and scope of community health workers be expanded to include high-impact but low-cost health and nutrition interventions. (The report found very little exclusive breastfeeding, with $51.3 \%$ of babies on mixed feeding.) Motsoaledi described infant milk formula as 'no different to skin lightening creams', vowing to help lead a charge at the World Health Assembly to have it banned altogether. He explained that with South Africa now aligned to best practice in ART and PMTCT, there was no longer any reason why the World Health Organization (WHO)'s recommendation for exclusive breastfeeding for the first 6 months shouldn't be followed.

\section{Gaps in HIV awareness campaigns}

The report highlighted the lack of HIV communication programmes in rural areas, and for English, Afrikaans, Tsonga and Venda speakers, recommending that future campaigns also focus more strongly on complementing school-based programmes and on children. It said an accreditation system should be introduced 'as a matter of urgency' to regularly monitor the quality of health facilities and to serve as a mechanism to hold managers accountable for the health outcomes of mothers and children.

Motsoaledi accepted the
criticisms and said he was
already prioritising the
restructuring of maternal
and child care units.

Motsoaledi accepted the criticisms and said he was already prioritising the restructuring of maternal and child care units. While $43 \%$ of maternal deaths were due to HIV, adverse incidents like antepartum and postpartum haemorrhage and sepsis cited in the report were due to 'basic protocols not being followed' and 'unacceptable'. When it was put to him by Izindaba that South Africa, having only $7 \%$ of the doctors required and $17 \%$ of nurses needed (by WHO standards), must contribute to the dismal quality of care, Motsoaledi again referred to quality assurance. 'Planning, development and the management of HR is on our 10-point plan but so often basic protocols are not followed, even in our teaching hospitals - we've left the basics behind.'

He agreed that the national accreditation of public health care facilities based on quality assurance for which the groundwork was currently being done by Dr Carol Marshal in his department, was a 'major priority'.

\section{Chris Bateman}

